\title{
Economic Consequences of Global Accounting Convergence: An Experimental Study of a Coordination Game
}

\author{
Satoshi Taguchi* Masayuki Ueeda Kazunori Miwa \\ Satoru Mizutani \\ Faculty of Commerce,
DOSHISHA UNIVERSITY \\ School of Business, \\ Graduate School of Economics, Faculty of Business Administration, \\ AOYAMA GAKUIN UNIVERSITY OSAKA UNIVERSITY \\ TEZUKAYAMA UNIVERSITY
}

\begin{abstract}
This paper examines whether the movement toward convergence of the International Financial Reporting Standards (IFRS) has ended. The methodology for this research is based on comparative institutional analysis and experimental game theory. In addition, we adopt a $3 \times 3$ coordination game because an essential factor of global accounting convergence is the coordination of accounting standards. The results confirm the termination of a movement toward the convergence of IFRS. Namely, our experiments indicate that global accounting convergence toward IFRS may not be successful. This is because some "egoist" countries maintain their initial systems and do not enter longterm cooperation.
\end{abstract}

JEL Classification: D74; F55; K22; M41; M48; P51

Key Words: NIA Approach; IFRS; Convergence; Coordination Game; Equilibrium Selection

\section{Introduction}

The globalization of trade and the rise of multinational corporations have led to the need for internationally harmonized accounting standards and practices to replace the disarray of varied and sometimes clashing national systems. To this end, the International Financial Reporting Standards (IFRS), developed and overseen by the IFRS Foundation and the International Accounting Standards Board (IASB), have thus far been adopted by numerous countries.

\footnotetext{
* Corresponding Author. AddressImadegawa-dōri Karasuma Higashi-Iru, Kamigyo-ku, Kyoto, JAPAN. Telephone +81 -75-251-3665E-mail staguchi@mail.doshisha.ac.jp This work was supported by JSPS KAKENHI Grant Number 23653118.
}

Received March 31, 2013; accepted October 7, 2013; available online December 9, 2013 (Advance publication by J-STAGE) DOI: 10.11640/tjar.3.2013.04 
This paper examines whether the movement toward convergence of IFRS has ended. According to IASB, their primary objective is

"To develop a single set of high quality, understandable, enforceable, and globally accepted IFRSs through its standard-setting body, the IASB. ${ }^{\prime}$

In other words, IASB is attempting to develop a sole accounting standard for the world and is a leading force behind convergence. Convergence thus far has gained strong global support, with over 100 countries including China and those in the EU having either adopted the standards in full or in part or planning to do in the future.

However, despite this support and a business case for such convergence, the harmonization process has run into problems. Neither the U.S. nor Japan, two economically powerful countries, have yet adopted IFRS while other countries have delayed implementation. Thus, the prospect of total global accounting standards convergence remains uncertain. This study focuses on the reasons for the failure of global accounting standards to converge toward "a single set of high quality, understandable, enforceable, and globally accepted IFRSs." These difficulties are in keeping with many other attempts at global coordinated action.

The methodology in this research is based on comparative institutional analysis (Aoki 2001; Grief 2006) and experimental game theory (Camerer 2003; Binmore 2010). In addition, we adopt a $3 \times 3$ coordination game because an essential factor of global accounting convergence is the coordination of accounting standards (Hail et al. 2010a, 2010b; Taguchi 2009).

We present the coordination game as a base model, derive its implications, and compare them with the results from experiments we conducted. Although the pure strategy Nash equilibrium of game theory anticipates global accounting convergence, our experiments indicate two possibilities: (1) convergence to only a current accounting system (e.g., the U.S. GAAP) rather than a new accounting system (e.g., IFRS) and (2) many countries adopt the "Stay" strategy whereby they maintain their own initial systems and do not cooperate with each other, thus becoming "egoists." ${ }^{2}$ We also show the theoretical background of the experimental results. Our results were supported by the equilibrium selection theory (Proposition 3) and a mixed strategy (Proposition 4 and Prediction).

The remainder of the paper is organized as follows. The next section discusses related literature. Sections 3 and 4 develop the model and explain the experimental design. Section 5 presents the experimental results. Section 6 offers a summery and concluding remarks.

\section{Related Literature}

\subsection{Arguments for and against convergence}

There are many arguments for and against IFRS (Hail et al. 2010a, 2010b). Some studies support convergence for the benefit of comparability ${ }^{3}$ of financial statements and network

\footnotetext{
1 The quotation is taken from the IASB website (http://www.ifrs.org/The+organisation/IASCF+and+IASB.htm).

2 In this paper, we define "egoists" (or an "egoist" player) as a subject who would like the other player to cooperate with $\mathrm{him} /$ her and adopts the "Stay" strategy (maintains his/her own initial system).

3 IASC (1989) lists "comparability" as an essential factor of financial statements.
} 
externalities (De Franco et al. 2009; Meeks and Swann 2009; Katz and Shapiro 1994). Other researchers oppose convergence and believe standard setting should be left to competition because the current standard setter is a monopoly (Dye and Sunder 2001). For example, Sunder (2002) has stated that competition helps create more efficient regulations. Although many arguments exist for and against the idea of convergence, minimal attention has been focused on conflict and coordination among countries regarding accounting standards. In addition, since the consequences of global convergence have not been predicted, this point remains open for discussion.

\subsection{Limitations of archival studies and needs of experimental studies}

Several empirical studies have examined the usefulness of IFRS (Barth et al. 2008; Daske et al. 2008), but they were only partial verifications that lacked cost-benefit analysis of adopting a single set of standards at the international level. Therefore, we experiment with artificial situations to discover more about such benefits and consequences.

The methodology in this research is based on comparative institutional analysis (Aoki 2001; Grief 2006) and experimental game theory (Camerer 2003; Binmore 2010). We test the predictions of game theory by experimentation.

\subsection{New institutional accounting approach}

This study also relates to the research framework for new institutional accounting (NIA), advocated by Wysocki (2011) who focuses on mutual dependence among various institutions, including those specializing in accounting. He stresses that financial reporting by enterprises is influenced by numerous institutional factors other than accounting standards, such as those related to law, auditing, capital market forces, ownership structures, and governance structures. Thus, in the current state of affairs in that many institutional factors differ by country and region, global convergence of accounting standards might have little essential meaning. This suggests that it will be difficult for IASB to achieve its goal of establishing a single set of globally accepted accounting standards. We address this issue by considering the convergence of accounting standards as a coordination problem among countries.

\subsection{Related models and their limitations}

Among earlier studies, Su and Guo (2006) and Fujii (2007) explained the convergence problem using a $2 \times 2$ coordination game. By assuming the existence of System A and System B, each player (player 1 or 2) decides which system to adopt. Each player is assumed to benefit by sharing his/her system (for example, the benefit in this case is assumed to be 1). Table 1 represents the payoff table for this game.

As is shown in Table 1, in their version of the game, the following two scenarios become pure-strategy Nash equilibria.

$($ Strategy of player 1 , Strategy of player 2$)=($ System A, System A $)$

$($ Strategy of player 1 , Strategy of player 2$)=($ System B, System B)

However, these express a game in which each player's first selection of a system is made under conditions in which neither had previously adopted any such system. This merely depicts a scenario in which a country that has not previously adopted any accounting standards selects 


\section{Table 1: Simple System Selection Game

\begin{tabular}{cccc}
\hline \hline & \multicolumn{2}{c}{ Player 2 } \\
\hline & & $\mathrm{A}$ & $\mathrm{B}$ \\
\hline Player 1 & $\mathrm{A}$ & 1,1 & 0,0 \\
& $\mathrm{~B}$ & 0,0 & 1,1 \\
\hline
\end{tabular}

Table 2: System Selection Game 2: When Each Player has Already Adopted a System at the Initial Stage

\begin{tabular}{cccc}
\hline \hline & \multicolumn{3}{c}{ Player 2 } \\
\hline & & $\mathrm{A}$ & $\mathrm{B}$ \\
\hline Player 1 & $\mathrm{A}$ & 3,2 & 1,1 \\
& $\mathrm{~B}$ & 0,0 & 2,3 \\
\hline
\end{tabular}

one for the first time and obtains benefits by coordinating with other countries. Therefore, a better fit for explaining the actual situation is a scenario that presupposes each player has already adopted a type of accounting standard (System A or B). In sum, the limitation of this model is the assumption of a situation in that no initial system exists.

Next, we expand this model and consider a scenario in which each player has already adopted a system at the game's initial stage. For example, player 1 adopts System A, while player 2 adopts System B. Moreover, the base state of each player (the benefit at the initial stage) is set as 1 . If players have previously adopted the same system as another player, they receive an additional benefit of 2. Moreover, the cost of changing systems is assumed to be 1 for each system. The payoff table in this case is represented in Table 2.

As is shown in Table 2, in this game, the following two scenarios become pure-strategy Nash equilibria.

$($ Strategy of player 1 , Strategy of player 2$)=($ System A, System A) $($ Strategy of player 1 , Strategy of player 2$)=($ System B, System B)

Moreover, it can be understood from these scenarios that adopting an action that coordinates with the system adopted by the other party is the best response for both players.

We must account for the fact that both players will obtain different benefits depending on whether the system is coordinated as System A or System B. More specifically, on the one hand, it is preferable for player 1, who originally adopted System A, to coordinate the whole system as A (i.e., player 2 selects A) than to coordinate it as B (player 1 changes his/her choice to System $\mathrm{B}$ to coordinate with player 2). On the other hand, for player 2, who originally adopted System $\mathrm{B}$, coordinating the whole system as B (i.e., player 1 selects $\mathrm{B}$ ) is preferable to coordinating it as A (player 2 changes his/her choice to System A to coordinate with player 1). When we consider this in the context of accounting standards, the above-mentioned scenarios indicate that the preferable situation for us is the one in which other countries coordinate its accounting standards to ours. In other words, because benefits accrue from coordinating accounting standards, adopting a strategy coordinated with the behavior of the other party becomes the mutually best response (therefore, the combination of such strategies becomes a Nash equilibrium). However, 
if we consider the costs required to switch systems, it is preferable for us if the other country coordinates its accounting standards with ours, rather than we give up our accounting standards and coordinate with another country's.

The above system-selection problem describes one where accounting standards already exist in each country. Namely, the expressed problem requires parties to choose to coordinate according to the accounting standards of one country or another. However, the actual problem for international accounting standards does not match the model of needing to coordinate accounting standards already adopted by one country or another. Rather, it is a question of whether to converge on new accounting standards (namely, the IFRS), or maintain the status quo. In other words, there is a need to envisage a coordination game that adds a "third choice" and create an appropriate model. Given this need, we present a new model that modifies the model used in previous research in the following aspects:

1. Introduction of an initial-period adoption system (introduction of system-change costs)

2. Introduction of a new "third choice"

Taguchi (2009) adopted these two aspects as a $3 \times 3$ coordination game. We use the Taguchi's (2009) model in the next section.

\section{Model}

The model of this study is based on the $3 \times 3$ coordination game developed in Taguchi (2009). This is because that model has two aspects (the initial system and a "third choice," discussed in section 2) and an essential factor of global accounting convergence is the coordination of accounting standards (Hail et al. 2010a, 2010b; Taguchi 2009).

Our model is a simple coordination game with players 1 and 2, each of whom represents a country ${ }^{4}$ that chooses a system from System A, B, and $\mathrm{N}$ as a strategy. ${ }^{5}$ It is assumed that Systems A and B have been respectively assigned to players 1 and 2 as their own initial systems and it entails a financial cost for each player to change the systems (Figure 1). When players adopt the same system, they each receive benefits from coordination (Figure 2), which include benefits from network externalities (Katz and Shapiro 1994) and the comparability of financial statements (IASC 1989).

Each player chooses one of three strategies: The first strategy for a player is to choose not to change the initial system (the "Stay" strategy), which we call $S$. The second strategy is to choose his/her partner (the other player) initially adopt (the "Other" strategy), which we call $O$.

\footnotetext{
${ }^{4}$ While enterprises as well as governments implement accounting standards and are more likely to operate across multiple nations, thereby facing varied accounting standard systems directly, our study uses states as the actors in our model for two reasons. First, states are ultimately the bodies with the legal power to determine and impose standards within their geographic region of authority. Second, enterprises are assumed to have avenues of representation within/to the state, so state action is assumed to take the interests of enterprises within its area into consideration.

${ }^{5}$ In reality, there are various levels of accounting convergence (see section 6). In this study, we simplify the model by considering only two possibilities, namely whether an accounting system has been adopted. This approach has been used in previous studies.
} 
Figure 1: SetTing (1)
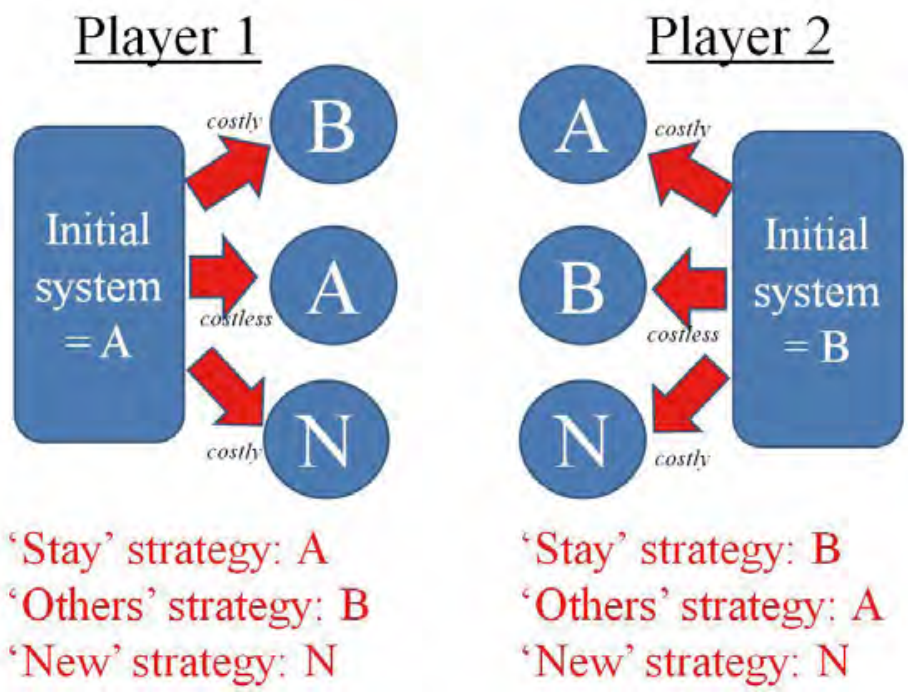

Figure 2: Setting (2)

When players adopt the same systems, they receive benefits for such coordination.

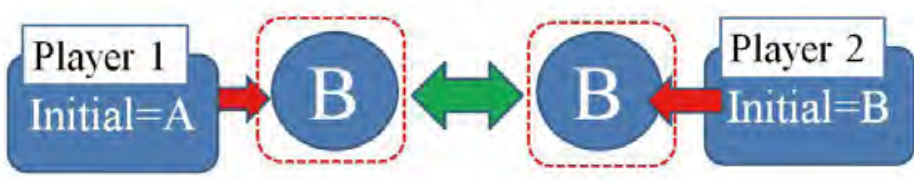

When players don't adopt the same systems, they don't receive benefits for such coordination.

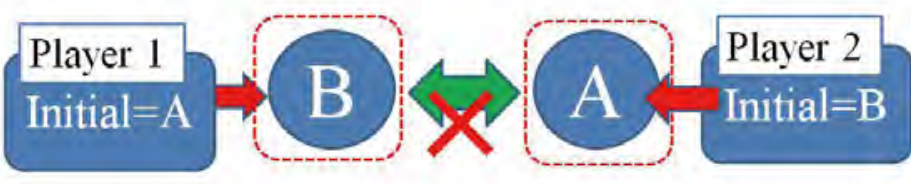

The third strategy is to choose the new system (the "New" strategy), which we call $N$.

The existence of each state's initial system and the costs to change them are the most important factors to consider when examining and undertaking institutional change. In reality, countries have pre-existing accounting standards, and making changes to such standards comes with a cost. These factors are known as institutional inertia (Aoki 2001) or a focal point (Schelling 1960).

We focus on the payoff function for players 1 and 2, shown in the following equation as benefit minus cost:

$$
\pi_{b}=b_{i j}-c_{b k}+T
$$



An Experimental Study of a Coordination Game

Table 3: Payoff Matrix

\begin{tabular}{|c|c|c|c|}
\hline & & Player 2 & \\
\hline & Stay & Other & New \\
\hline $\begin{aligned} \text { Player } 1 & \text { Stay } \\
& \text { Other } \\
& \text { New }\end{aligned}$ & $\begin{array}{c}\mathrm{T}, \mathrm{T} \\
b_{O S}-c_{1 O}+T, b_{O S}+T \\
-c_{1 N}+T, \mathrm{~T}\end{array}$ & $\begin{array}{c}b_{S O}+T, b_{S O}-c_{2 O}+T \\
-c_{1 O}+T,-c_{2 O}+T \\
-c_{1 N}+T,-c_{2 O}+T\end{array}$ & $\begin{array}{c}\mathrm{T},-c_{2 N}+T \\
-c_{1 O}+T,-c_{2 N}+T \\
b_{N N}-c_{1 N}+T, b_{N N}-c_{2 N}+T\end{array}$ \\
\hline
\end{tabular}

Here, $T$ is the endowment for each player. The benefit of coordination is shown as $b_{i j}$, which is derived by comparability and network externalities. In addition, it benefits player $b$ when player 1 adopts strategy $i(i=S, O, N)$ and player 2 adopts strategy $j(j=S, O, N)$. Denote by $c_{b k}$ the cost to change systems when player $h$ chooses strategy $k(k=S, O, N)$. We assume costs as follows:

$$
\begin{gathered}
b_{S O}>0, b_{O S}>0, b_{N N}>0, b_{S S}=b_{O O}=b_{S N}=b_{N S}=b_{O N}=b_{N O}=0 . \\
c_{1 S}=c_{2 S}=0, c_{1 O}>0, c_{2 O}>0, c_{1 N}>0, c_{2 N}>0 .
\end{gathered}
$$

The payoff matrix is shown in Table 3.

\subsection{Equilibrium (1): dilemma of IFRS}

In this subsection, we run our model using several variations of the costs and the benefits for each actor that will weight their decisions in different directions. There are four variations.

The first is the base model that meets three conditions:

(1) Each benefit is at the same level $\left(b_{S O}=b_{O S}=b_{N N}=\bar{b}\right)$.

(2) Each cost is at the same level $\left(c_{1 O}=c_{2 O}=c_{1 N}=c_{2 N}=\bar{c}\right)$.

(3) Each benefit exceeds each cost $(\bar{b}-\bar{c}>0)$.

In the second variation, each cost is at the same level $\left(c_{1 O}=c_{2 O}=c_{1 N}=c_{2 N}=c\right)$, but each benefit differs. The third variation has each benefit at the same level $\left(b_{S O}=b_{O S}=b_{N N}=b\right)$, but each cost differs. The fourth variation sees both benefits and costs differing.

As the first step in our analysis, we examine the base model. Table 4 shows the payoff matrix for the base model.

In addition, Table 5 shows the payoff matrix for the base model in the simple case of $T=1, \bar{b}=2, \bar{c}=1$.

A pair of strategies in a two-player game is defined to be Pareto optimal if there is no other pair of strategies that strictly increase the payoff of one player without decreasing that of the other player, and equitable if the associated payoffs of the two players are equal. The following proposition shows properties of Nash equilibria in pure strategies of the game.

Proposition 1. Nash equilibrium and its characteristics (Taguchi, 2009).

The Nash equilibria in pure strategies are:

(Strategy of player 1, Strategy of player 2) $=(S, O),(O, S),(N, N)$.

The Nash equilibria $(S, O)$ and $(O, S)$ are Pareto optimal but are not equitable. On the other hand, the Nash equilibrium $(N, N)$ is equitable but not Pareto optimal. 
Table 4: Payoff Matrix of the Base Model

\begin{tabular}{lcccc}
\hline \hline \multicolumn{4}{c}{ Player 2 } \\
\hline Player 1 1 Stay & T, T & Other & New \\
& Other & $\bar{b}-\bar{c}+T, \bar{b}-\bar{c}+T$ & $\mathrm{~T},-\bar{c}+T$ \\
& New & $-\bar{c}+T, T$ & $-\bar{c}+T,-\bar{c}+T$ & $-\bar{c}+T,-\bar{c}+T$ \\
& & $-\bar{c}+T,-\bar{c}+T$ & $\bar{b}-\bar{c}+T, \bar{b}-\bar{c}+T$ \\
\hline
\end{tabular}

Table 5: Payoff Matrix of the Base Model in Which $T=1, \bar{b}=2, \bar{c}=1$

\begin{tabular}{llccc}
\hline \hline & & & Player 2 \\
\hline & & Stay & Other & New \\
\hline Player 1 1 & Stay & 1,1 & 3,2 & 1,0 \\
& Other & 2,3 & 0,0 & 0,0 \\
& New & 0,1 & 0,0 & 2,2 \\
\hline
\end{tabular}

As seen in Proposition 1, this case includes multiplicity of equilibria, wherein equilibrium is not specifically determined. This proposition presents two implications for global accounting convergence.

First, there are three patterns of " $a$ single set of accepted international financial reporting standards" by the IASB. There is no natural result wherein countries worldwide adopt a particular accounting system a priori, although IASB aims at the Nash equilibrium $(N, N)$.

Second, the proposition also shows the relationship among the three equilibria, in which trade-offs between equity and efficiency exist. We call this trade-off "the first dilemma of IFRS." Although IASB aims for the equilibrium $(N, N)$, it is not Pareto optimal but "fair." In addition, we may suppose that the player who aims for equilibrium $(S, O)$ or $(O, S)$ is the U.S. For example, it appears that the U.S. Securities and Exchange Commission aims for convergence to Statements of Financial Accounting Standards since the payoff for the U.S. would be higher than that for other countries.

\subsection{Equilibrium (2): Mixed strategy Nash equilibrium}

In this subsection, we solve the mixed extension of the same game. The following proposition shows the existence of a mixed strategy Nash equilibrium other than the Nash equilibria in pure strategies of the game.

Proposition 2. Mixed strategy Nash equilibrium of the base model.

For players 1 and 2 to choose $S$ with probability 50\% and $N$ with probability 50\% is a mixed strategy Nash equilibrium of the base model.

Proof. See Taguchi (2012).

This proposition implies that the state $(N, N)$ may be attained even though it is not Pareto optimal and there are "Other" Pareto optimal states. We call this "the second dilemma of IFRS." 


\subsection{Hypotheses}

We propose the following hypotheses for the base model:

Hypothesis 1 (based on the first dilemma of IFRS).

H1-1. Each player adopts each of the strategies $S, O$, and $N$ with the same probability.

H1-2. Each of the strategy profiles $(S, O),(O, S)$, and $(N, N)$ is realized with the same probability.

Hypothesis 2 (based on the second dilemma of IFRS).

H2-1. Each player adopts both $S$ and $N$ with the same probability.

$H 2-2$. Only $(N, N)$ is realized.

\section{Experimental design}

To explain our experimental design, we again use the case in which $T=1, \bar{b}=2$, and $\bar{c}=1$ (Table 5).

We report data from 34 subjects shown in the design table 6, collected in two separate sessions, with 14-20 subjects typically participating in each session.

All sessions were conducted at Doshisha University in Japan. Subjects were primarily undergraduate students from Doshisha University recruited by advertisements and e-mail. Due to the abstract and relatively simple nature of the decision task, a background in accounting was not a prerequisite for participation. No one participated in more than one session of this experiment. Each participant took part in 20 rounds of decision making. ${ }^{6}$ Participants took about 90 minutes (including instructions) to complete and earned about $¥ 2,578$ on average.

The experiment was run on networked computers using the $\mathrm{z}$-Tree experiment software package (Fischbacher 2007) (Figure 3). Our $3 \times 3$ matrix model is a single-period one in which both nations are assumed to simultaneously decide and cannot interact with each other. These assumptions may hinder them from choosing more co-operational decisions. However, as Licht (1999, 82) suggested, understanding how a single-period model works in the laboratory is important for clarifying the underlying conflict that requires reconciliation. Therefore, our subjects were asked not to directly communicate with one another, and the only interactions were via a computer program. Also, all treatments randomly re-matched the pairs of subjects in each period to minimize the potential impact of reciprocal concerns.

At the beginning of a session, each subject separated by partitions in a laboratory and given written instructions. Instructions were also read aloud to subjects in an attempt to make the rules of the game common knowledge. After this, subjects took a quiz to ensure they understood them. We strove for neutral terminology in the instructions.

After action choices were entered, each subject was shown the following information: his/her own action, another's action, and his/her own payoff (Figure 4). In all treatments, subjects were not given information, either individually or in aggregate, about the results of other

\footnotetext{
${ }^{6}$ We know that the change in accounting standards has been slow and that once nations have switched their standards it would be difficult to undo. However, conventional wisdom insists that subjects adjust their strategies with replications and convergence to equilibrium. We address whether and how equilibrium is achieved in the next section.
} 
Table 6: Characteristics of Subjects (Base Model)

\begin{tabular}{|c|c|c|c|c|c|c|}
\hline Session & Date & Place & Subjects & Male & Female & Average age \\
\hline 1 & Nov 25, 2011 & Doshisha University & 14 & 7 & 7 & 21.7 \\
\hline 2 & June 18,2012 & Doshisha University & 20 & 13 & 7 & 20.9 \\
\hline & & & 34 & 20 & 14 & 21.2 \\
\hline
\end{tabular}

Figure 3: Z-TREe

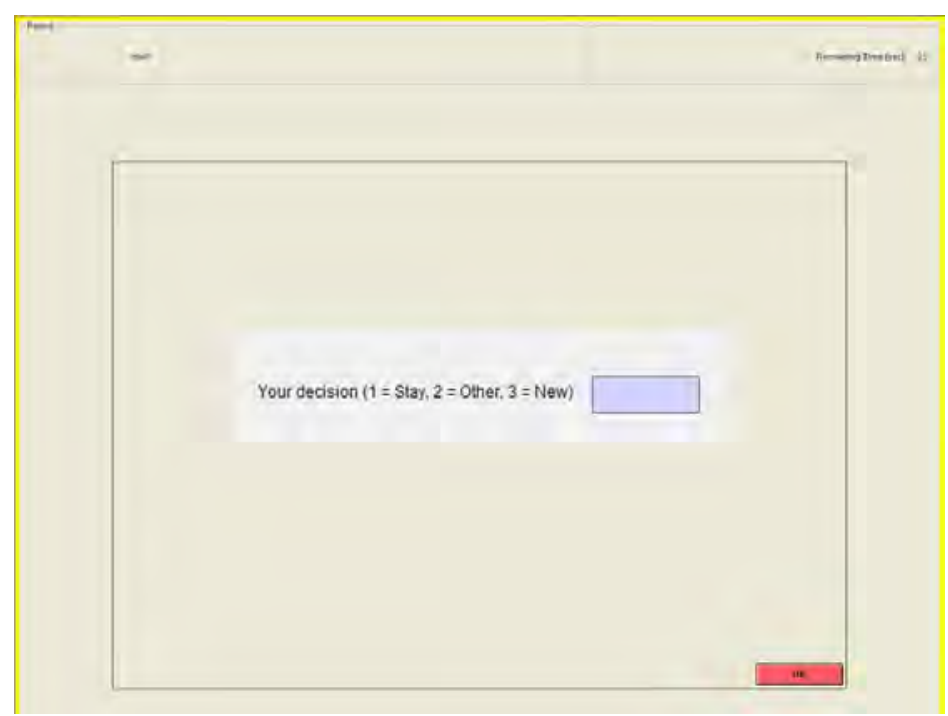

Figure 4: Information After Action Choices were Entered

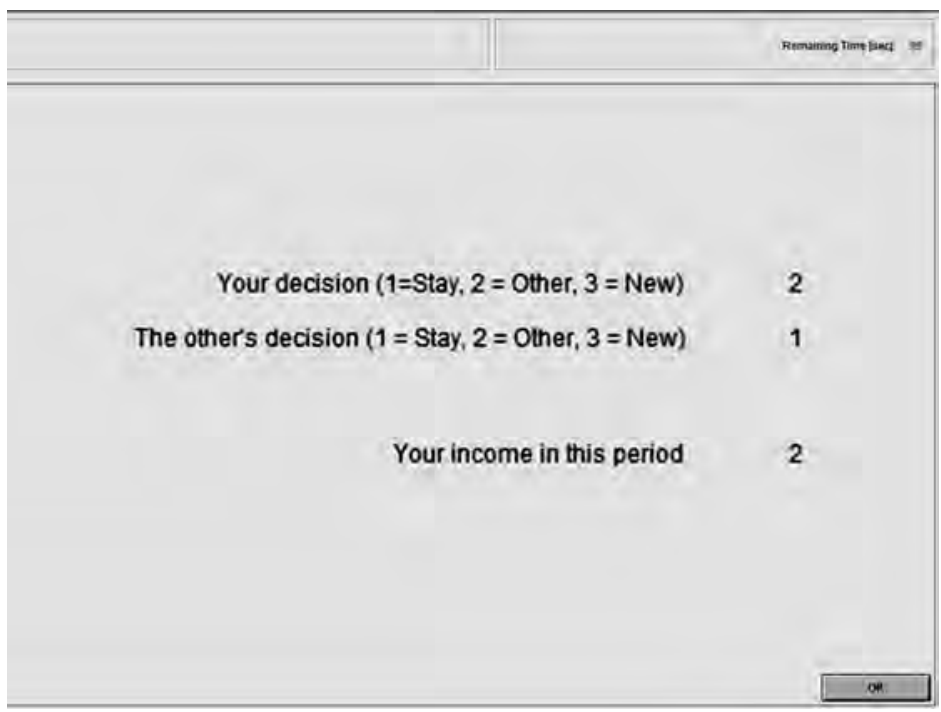


pairs of subjects. At the end of each round, subjects were asked to observe their results and enter the information from that round into a record sheet.

The experimental session ended after round 20. Each subject received his/her earnings from 20 rounds at the rate of $¥ 50$ per point. Additionally, all subjects received $¥ 1,000$ for showing up.

\section{Results of the Base Model Experiment}

\subsection{Personal decision: rates of strategies that subjects adopted}

In total, 34 subjects participated, and each played 20 rounds, producing 680 observations. To show the results of the experiment, we focus on the rates of the strategies that subjects adopted, as shown in Table 7 and Figure 5.

Contrary to our hypotheses, results clearly indicated that subjects tended either to maintain their initial system or to adopt their partner's system, but not System N. The probability that subjects adopted System $\mathrm{N}$ was significantly lower according to the chi-square test (1\%). The results rejected $\mathrm{H} 1-1$ and $\mathrm{H}$ 2-1.

Next, we focus on these rates in a time series. As seen in Figure 6, the results clearly indicate that subjects tend to first maintain their initial system and then adopt their partner's system. From rounds 1-20, the probabilities of adopting the "Stay" strategy (maintain their initial system) and adopting the "Other" strategy (adopt their partner's system) were high.

\subsection{Matching: rates of the combination of strategies}

As seen in Table 8 and Figure 7, the percentage of Pareto optima $(S, O)$ and $(O, S)$ in the observations was $47.35 \%$ and that of the equitable Nash equilibrium $(N, N)$ was $0 \%$. This result is surprising. The concept of Nash equilibrium predicts that $(N, N)$ would be realized, but the experimental results rejected $\mathrm{H} 1-2$ and $\mathrm{H} 2-2$.

This finding demonstrates our first implication for global accounting convergence. Theoretically, there are two possible economic consequences of global accounting convergence. First, we have three possible patterns of "a single set of accepted IFRS": $(S, O),(O, S)$, and $(N$, $N)$. We have named this "the first dilemma of IFRS." Second, using the concept of mixed strategy, we may have only one pattern of "a single set of accepted IFRS": $(N, N)$. We have named this "the second dilemma of IFRS."

In our experiment data, there were many observations of the unequitable Nash equilibria $(S$, $O)$ and $(O, S)$. On the other hand, there was no observation of the equitable Nash equilibrium $(N, N)$ although IASB currently plans a convergence to $(N, N)$.

The literature offers two explanations of why countries might spurn convergence despite the benefits. One type, which we call the "egoists," is defined as a subject who would like the other player to cooperate with him/her and adopts the "Stay" strategy (maintains his/her own initial system). The U.S. would be categorized into this type. The other type, which we call the "generous," is represented by Japan. Such two countries would attain the "unfair" equilibria of $(S$, $O)$ and $(O, S)$. Therefore, we return to our main research question: Will the movement toward convergence of IFRS finally end? The answer is "Yes." The termination will be confirmed if IASB plays a central role in the convergence and it is the current style under observation. There will be another story of global convergence if the U.S. is central to the convergence according 
Table 7: Rates of the Strategies that Subjects Adopted (Base Model Experiment)

\begin{tabular}{lrrrc}
\hline \hline & S (Stay) & O (Other) & N (New) & Total \\
\hline Sample Number & 427 & 243 & 10 & 680 \\
Rate (\%) & 62.79 & 35.74 & 1.47 & 100 \\
\hline
\end{tabular}

Figure 5: Rates of the Strategies that Subjects Adopted

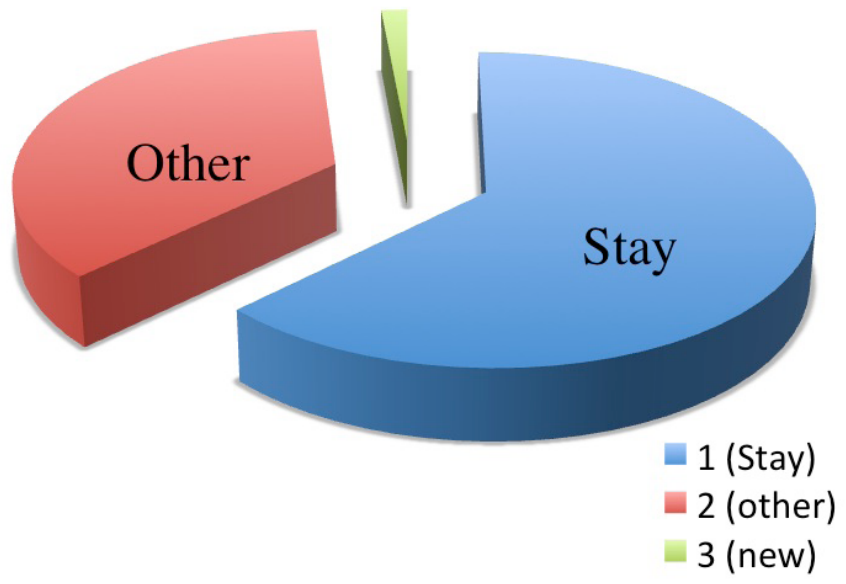

Figure 6: Rates of the Strategies that Subjects Adopted in Time Series

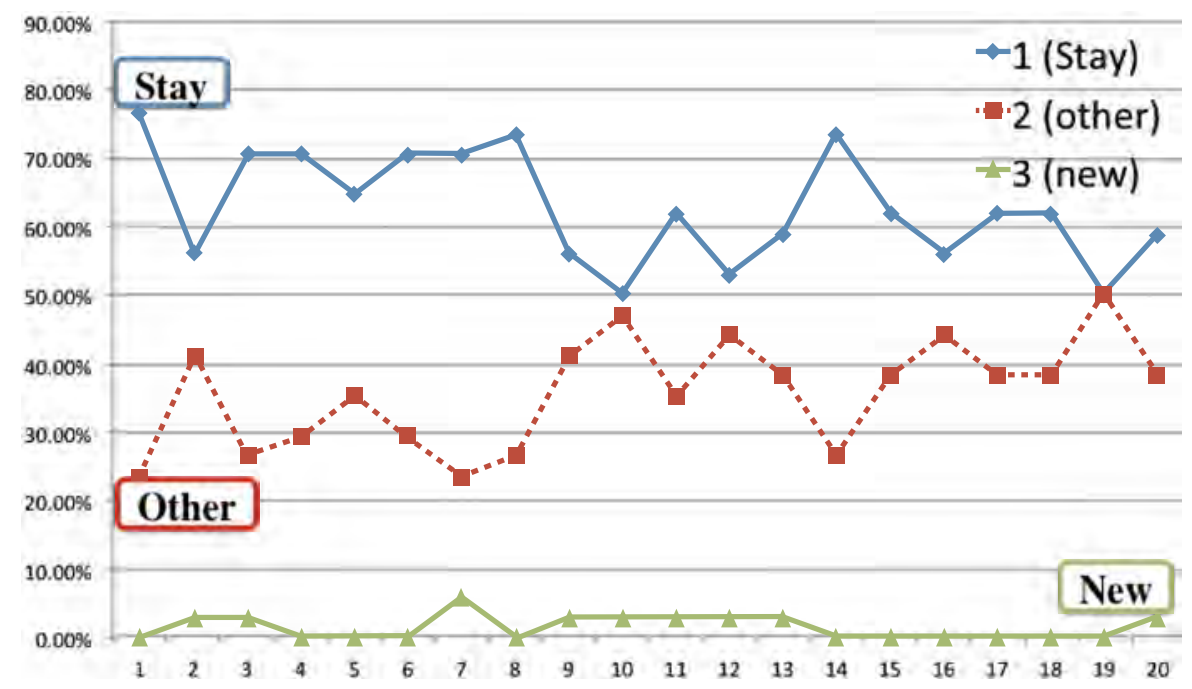

with the "unfair" equilibria of $(S, O)$ and $(O, S)$.

In addition, the percentage of $(S, S)$ was relatively high (38.82\%). This strategy profile represents that players 1 and 2 respectively adopt Systems A and B, each of which is the initial systems of the player that chooses. This means that each subject did nothing by him/herself and wanted the other player (his/her partner) to change his/her initial system. This result is also contrary to the prediction of Propositions 1 and 2.

This result produces our second implication for global accounting convergence. Theoretically, 
Table 8: Rates of the Combinations of Strategies (Base Model Experiment)

\begin{tabular}{lrrrrrrr}
\hline \hline & SS & SO/OS & SN/NS & OO & ON/NO & NN & Total \\
\hline Sample Number & 132 & 161 & 4 & 37 & 6 & 0 & 340 \\
Rate (\%) & 38.82 & 47.35 & 1.18 & 10.88 & 1.76 & 0.00 & 100 \\
\hline
\end{tabular}

Figure 7: Rates of the Combinations of Strategies in Time Series

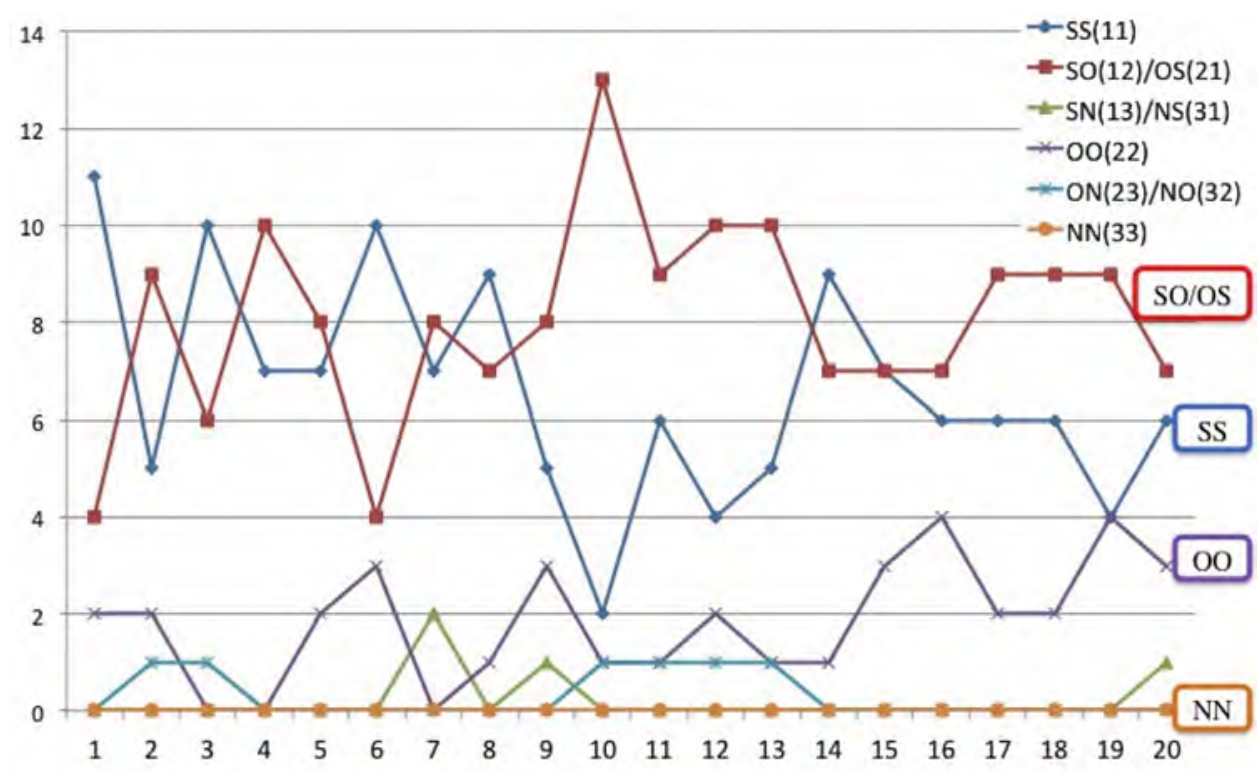

neither player will adopt the Stay strategy. Contrary to this theoretical prediction, however, our experiment revealed that there is a high possibility that the strategy combination of $(S, S)$ will be adopted. With reference to accounting coordination, therefore, the results of our experiments indicate the likelihood that countries will do nothing and maintain their initial systems. This raises the question: Why was the rate of the combination of $(S, S)$ higher? One possible explanation is "institutional inertia." To achieve the equilibrium of $(N, N)$, all players must cooperate. However, if one party betrays such cooperation, the other will bear the cost and receive no overall benefit (i.e., a net loss). As a result, the losing party will have no incentive to cooperate. In other words, if all players become "egoists," coordination will be impossible. ${ }^{7}$

\subsection{Theoretical background of the result}

The experimental results did not support Hypothesis 1 or 2 .

To analyze this gap between theoretical and actual outcomes, we will examine two factors. The first factor is the equilibrium selection from the three pure strategy Nash equilibria. The incidence rate of the two equilibria $(S, O)$ and $(O, S)$ was $47.35 \%$, and that of the other equilibrium $(N, N)$ was $0 \%$. We consider why $(S, O)$ and $(O, S)$ were achieved and $(N, N)$ was eliminated. The second factor is the likelihood of $(S, S)$. We investigate why its incidence rate was so high in spite of the fact that $(S, S)$ is not a Nash equilibrium in pure strategies.

\footnotetext{
7 We explain more theoretically why the rate of the combination of $(S, S)$ is so high (section 5.3).
} 


\subsubsection{Equilibrium selection problem}

First, the equilibrium selection problem will be discussed. This game is constructed from multiple Nash equilibria. An equilibrium selection theory, which can be employed to determine the selection of an equilibrium, is required to resolve this problem of multiple equilibria (Harsanyi and Selten 1988). There are two important concepts in the equilibrium selection theory: payoff dominance and risk dominance.

Payoff dominance asserts that among multiple equilibria, the one that payoff dominates will be selected. Risk dominance asserts that among multiple equilibria, the equilibrium point having a large player's deviation loss product will dominate. Propositions such as those described below can be specifically derived on the basis of the base model.

Proposition 3. Payoff-dominance Equilibrium and Risk-dominance Equilibrium in the Base Model

Of the multiple equilibria in the base model, no payoff-dominance equilibrium exists. The riskdominance equilibria are $(S, O)$ and $(O, S)$.

Proof. See Appendix.

From the perspective of Proposition 3, the equilibria $(S, O)$ and $(O, S)$ are chosen because of risk dominance. This is consistent with our experimental results.

\subsubsection{Why was the rate of $(S, S)$ so high even though $(S, S)$ was not a Nash equilibrium?}

In this subsection, we discuss why subjects selected a strategy profile other than a Nash equilibrium. In the experiment, the incidence rate of $(S, S)$, which is not a Nash equilibrium in pure strategies, was very high (38.82\%). We carefully examine a reason for that result. The key concept used here is a mixed strategy Nash equilibrium. More specifically, Proposition 2 stated that there was a Nash equilibrium in mixed strategies of the base game. Moreover, this game has another mixed strategy Nash equilibrium.

Proposition 4. Mixed strategy Nash equilibrium of the base model.

For players 1 and 2 to choose $S$ with probability 75\% and $N$ with probability 25\% is a mixed strategy Nash equilibrium of the base model.

Proof. See Appendix.

When the types of strategy combinations that arise together are calculated on the basis of Proposition 4, the following prediction can be made.

Prediction. The incidence rates of strategy profiles

$$
(S, O) \text { or }(O, S),(S, S) \text {, and }(O, O)
$$

in the base model are respectively

$6 / 16(37.5 \%), 9 / 16(56.25 \%)$, and $1 / 16(6.25 \%)$. 
The above-mentioned prediction is consistent with the experimental results in which there were many observations of $(S, S)$ and no observation of $(N, N)$.

\section{Conclusion}

We have examined whether the movement of global convergence toward IFRS reaches an end. The main implication is derived from an experiment of the base model. Although the concept of pure strategy Nash equilibrium of game theory predicts global accounting convergence, the results from our experiment indicate two alternate possibilities: (1) the global accounting converges to an existing system (e.g., the U.S. GAAP) rather than a new system (e.g., IFRS) and (2) many countries maintain their own initial systems and do not cooperate with each other (we have named such countries "egoists"). We have also found that our experimental results were supported by two other concepts of game theory. One is risk-dominance (Proposition 3). The other is mixed strategy Nash equilibrium (Proposition 4 and Prediction). By employing a simple coordination game, our study provides a first step toward a better understanding of the failure of global accounting standards to fully converge.

Some issues to be discussed in the future are as follows. First, one can develop our coordination model by incorporating additional options such as changes in strategies based on the concept of Nash equilibrium as we did. Second, one can extend our coordination model to a sequential-decision game in which each player sequentially makes a decision, or to a signaling game in which players have a communication tool. Third, one can investigate factors or mechanisms that stabilize global accounting convergence. For example, Taguchi (2012) proposes a correlated equilibrium model for the stabilization.

\section{Appendix}

\section{A.1 Proof of Proposition 3}

We define payoff dominance and risk dominance. We denote the set of pure strategies for player $i$ by $S_{i}$, - i.e., $S_{i}=\{S, O, N\}$, where $S=$ "Stay," $O=$ "Other," and $N=$ "New." The payoff function of player $i$ is $u_{i}: \mathrm{S}_{1} \times S_{2} \rightarrow \mathbb{R}$. Suppose $\left(s_{1}^{*}, s_{2}^{*}\right),\left(s_{1}^{\prime}, s_{2}^{\prime}\right) \in S_{1} \times S_{2}$ are Nash equilibria. Nash equilibrium $\left(s_{1}^{*}, s_{2}^{*}\right)$ payoff dominates $\left(s_{1}^{\prime}, s_{2}^{\prime}\right)$ if $u_{i}\left(s_{1}^{*}, s_{2}^{*}\right)>u_{i}\left(s_{1}^{\prime}, s_{2}^{\prime}\right)$ for player $i=1$, 2 . In contrast, Nash equilibrium $\left(s_{1}^{*}, s_{2}^{*}\right)$ risk dominates $\left(s_{1}^{\prime}, s_{2}^{\prime}\right)$ if $\left\{u_{1}\left(s_{1}^{*}, s_{2}^{*}\right)-u_{1}\left(s_{1}^{\prime}, s_{2}^{*}\right)\right\}\left\{u_{2}\left(s_{1}^{*}, s_{2}^{*}\right)-\right.$ $\left.u_{2}\left(s_{1}^{*}, s_{2}^{\prime}\right)\right\}>\left\{u_{1}\left(s_{1}^{\prime}, s_{2}^{\prime}\right)-u_{1}\left(s_{1}^{*}, s_{2}^{\prime}\right)\right\}\left\{u_{2}\left(s_{1}^{\prime}, s_{2}^{\prime}\right)-u_{2}\left(s_{1}^{\prime}, s_{2}^{\prime}\right)\right\} .8$

We consider our base model. By following the above definition, we find no payoff dominance relationship among $(S, O),(O, S)$, and $(N, N)$. On the other hand, $(S, O)$ risk dominates $(N, N)$ because:

$$
\begin{gathered}
\left\{u_{1}(S, O)-u_{1}(N, O)\right\}\left\{u_{2}(S, O)-u_{2}(S, N)\right\}=(3-0)(2-0)=6 \\
>\left\{u_{1}(N, N)-u_{1}(S, N)\right\}\left\{u_{2}(N, N)-u_{2}(N, O)\right\}=(2-1)(2-0)=2 .
\end{gathered}
$$

${ }^{8}$ See, Harsanyi and Selten (1988). 
By a similar procedure, we find that $(O, S)$ also risk dominates $(N, N)$ and that there is no risk dominance relationship between $(S, O)$ and $(O, S)$.

\section{A.2 Proof of Proposition 4}

We denote a mixed strategy for player $i$ by $\sigma_{i}: S_{i} \rightarrow[0,1]$, which assigns to each pure strategy $s_{i} \in S_{i}$ a probability $\sigma_{i}\left(s_{i}\right) \geq 0$. We also let $\sigma=\left(\sigma_{1}, \sigma_{2}\right)$. Note that a necessary and sufficient condition for mixed strategy profile $\sigma$ to be a Nash equilibrium is that each player, given the distribution of strategies played by his opponent, is indifferent among all the pure strategies that he plays with positive probability and that these pure strategies are at least as good as any pure strategy he plays with zero probability. ${ }^{9}$

We focus on strictly mixed strategy equilibria and restrict the analysis to those that are symmetrical. Suppose player 2 randomizes between "Stay" and "Other." That is, player 2 plays "Stay" with probability $q$, "Other" with probability $(1-q)$, and "New" with zero probability, i.e., $\sigma_{2}=(q, 1-q, 0)$ where $q \in(0,1)$. Then, in a Nash equilibrium, player 2 must be indifferent between "Stay" and "Other," and his expected payoff from playing "New" must not exceed that from playing "Stay" or "Other." Furthermore, player 1 best responds to player 2's strategy. Player 1's expected payoffs from playing "Stay," "Other," and "New" are as follows:

$$
\begin{gathered}
\mathrm{E} u_{1}\left(S, \sigma_{2}\right)=1 q+3(1-q)=3-2 q, \\
\mathrm{E} u_{1}\left(O, \sigma_{2}\right)=2 q+0(1-q)=2 q, \\
\mathrm{E} u_{1}\left(N, \sigma_{2}\right)=0 q+0(1-q)=0
\end{gathered}
$$

where $\mathrm{E}$ is the expectation operator. When $q>3 / 4(q<3 / 4)$, player 1's best response is to play the pure strategy "Other" ("Stay"). However, if so, then player 2 cannot obtain the same expected payoffs from playing "Stay" and "Other," violating the indifference condition above. In contrast, if $q=3 / 4$, then player 1 also plays "Stay" and "Other" with positive probabilities. Thus, given the symmetrical structure of the game, we find that $\sigma^{*}=((3 / 4,1 / 4,0),(3 / 4,1 / 4,0))$ is a candidate for reaching Nash equilibrium.

To check whether the mixed strategy profile $\sigma^{*}$ is a Nash equilibrium, we calculate player $i$ 's expected payoffs given that player $j$ plays the mixed strategy $\sigma_{j}^{*}=(3 / 4,1 / 4,0)$ :

$$
\begin{gathered}
\mathrm{E} u_{i}\left(S, \sigma_{j}^{*}\right)=3-2(3 / 4)=3 / 2 \\
\mathrm{E} u_{i}\left(O, \sigma_{j}^{*}\right)=2(3 / 4)=3 / 2 \\
\mathrm{E} u_{i}\left(N, \sigma_{j}^{*}\right)=0 .
\end{gathered}
$$

Therefore, each player is indifferent between "Stay" and "Other" (played with positive probabilities) and the expected payoff from playing "New" (played with zero probability) is not greater than that from playing "Stay" and "Other." Therefore, we conclude that the mixed

\footnotetext{
${ }^{9}$ See Mas-Colell et al. (1999, Proposition 8.D.1, 250).
} 
strategy profile $\sigma^{*}=((3 / 4,1 / 4,0),(3 / 4,1 / 4,0))$ is a Nash equilibrium.

By applying similar reasoning to other mixed strategy profiles, we find that $((1 / 2,0,1 / 2)$, $(1 / 2,0,1 / 2))$ is also a Nash equilibrium. ${ }^{10}$

\section{REFERENCES}

AoKI, M. 2001. Towards a Comparative Institutional Analysis. Cambridge, MA: The MIT Press.

BARTH, M., W. R. LANDSMAN, AND M. H. LANG. 2008. International accounting standards and accounting quality. Journal of Accounting Research 46 (3): 467-498.

Binmore, K. 2010. Game theory and institution. Journal of Comparative Economics 38 (3): 245-252.

Camerer, C. F. 2003. Behavioral Game Theory: Experiments in Strategic Interaction. Princeton, NJ: Princeton University Press.

Daske, H., L. Hail, C. LeuZ, AND R. Verdi. 2008. Mandatory IFRS reporting around the world: Early evidence on the economic consequences. Journal of Accounting Research 46 (5): 1085-1142.

De Franco, G., S. Kothari, and R. Verdi. 2009. The benefits of financial statement comparability. Working paper, MIT Sloan School of Management.

Dye, R. A. AND S. Sunder. 2001. Why not allow FASB and IASB standards to compete in the U.S.? Accounting Horizons 15 (3): 257-271.

Fischbacher, U. 2007. z-Tree: Zurich Toolbox for ready-made economic experiments. Experimental Economics 10 (2): 171-178.

FujII, H. 2009. A Study on an Accounting for Institutional Change. Tokyo, JAPAN: Chuokeizai-Sha (in Japanese).

Grief, A. 2006. Institutions and the Path to the Modern Economy: Lessons from Medieval Trade. Cambridge: Cambridge University Press.

Hail, L., C. LeuZ, ANd P. Wysocki. 2010a. Global accounting convergence and the Potential Adoption of IFRS by the U.S. (Parts 1): Conceptual Underpinnings and Economic Analysis. Accounting Horizons 24 (3): 355-394.

Hail, L., C. LeuZ, And P. Wysocki. 2010b. Global accounting convergence and the Potential Adoption of IFRS by the U.S. (Parts 2): Political Factors and Future Scenarios for U.S. Accounting Standards. Accounting Horizons 24 (4): 567-588.

Harsanyi, J. C. and R. Selten. 1988. A General Theory of Equilibrium Selection in Games. Cambridge, MA: The MIT Press.

IASC. 1989. Framework for the Preparation and Presentation of Financial Statements.

Katz, M. L. AND C. Shapiro. 1994. Systems competition and network effects. Journal of Economic Perspectives 8 (2): 93-115.

Licht, A. N. 1999. Games commissions play: $2 \times 2$ Games of international securities regulation. The Yale Journal of International Law 24: 61-125.

Mas-Colell, A., M. D. Whinston, and J. R. Green. 1999. Microeconomic Theory. Oxford: Oxford University Press.

Meeks, G., And G. Swann. 2009. Accounting standards and the economics of standards. Accounting and Business Research 39 (3): 191-210.

Schelling, T. C. 1960. The Strategy of Conflict. Cambridge MA: Harvard University Press.

Su, T.-C. And X.-M. Guo. 2006. An analysis of globalization of accounting standards based on game theory. Journal of Modern Accounting and Auditing 2: 15-22.

SundER, S. 2002. Regulatory competition among accounting standards within and across international boundaries. Journal of Accounting and Public Policy 21 (3): 219-234.

${ }^{10}$ A referee pointed out that two asymmetric equilibria exist in the base game, which are $((1 / 2,0,1 / 2),(0,1 / 4,3 / 4))$ and $((0,1 / 4,3 / 4),(1 / 2,0,1 / 2))$. 
TAGUCHI, S. 2009. Will the movement towards convergence of international financial reporting standards finally come to an end? Doshisha Shogaku 61 (3): 24-46 (in Japanese).

TAGUCHI, S. 2012. A study on the global accounting convergence by meta-rule: Institution and correlated equilibrium. Doshisha Shogaku 63 (5): 98-120 (in Japanese).

WysOcki, P. 2011. New institutional accounting and IFRS. Accounting and Business Research 41 (3): 309328. 